Mots. Les langages du politique

70 | 2002

La politique en chansons

\title{
Chants de liberté et de solidarité, pour la Grèce et la Pologne
}

Songs of freedom and solidarity in sympathy with Greece and Poland

Cantos de libertad y de solidaridad por Grecia y Polonia

Frédérique Tabaki-lona

\section{OpenEdition}

Journals

Édition électronique

URL : https://journals.openedition.org/mots/9193

DOI : $10.4000 /$ mots. 9193

ISSN : 1960-6001

Éditeur

ENS Éditions

Édition imprimée

Date de publication : 1 novembre 2002

Pagination : 45-63

ISBN : 2-84788-016-X

ISSN : 0243-6450

\section{Référence électronique}

Frédérique Tabaki-Iona, "Chants de liberté et de solidarité, pour la Grèce et la Pologne », Mots. Les

langages du politique [En ligne], 70 | 2002, mis en ligne le 25 mars 2011, consulté le 23 avril 2022. URL: http://journals.openedition.org/mots/9193 ; DOl : https://doi.org/10.4000/mots.9193

\section{(c) ENS Éditions}


Frédérique TABAKI-IONA ${ }^{\circ}$

\section{Chants de liberté et de solidarité, pour la Grèce et la Pologne}

Les sentiments de sympathie des Français envers la Grèce et la Pologne s'insèrent dans un large courant de solidarité envers les autres peuples d'Europe. Le philhellénisme, selon les poètes et les chansonniers, sert d'exemple lumineux au mouvement polonophile qui lui succède. On pourrait dire qu'il s'agit d'un vaste élan européen de romantisme social libérateur qui s'exprime, sur un demi-siècle, à travers poésies et chansons.

C'est sous la Restauration, pendant la lutte pour son indépendance menée par la Grèce asservie sous le joug ottoman (1821-1828), que le mouvement philhellénique s'inspire de cette cause et s'empare d'une société française sensible aux évènements tragiques qui jalonnent cette guerre. Seule une aide de la part de l'Europe pouvait empêcher l'anéantissement des insurgés par les Turcs. Entrainée, avec l'Angleterre, à l'avant-garde de ce qui se passe dans d'autres nations européennes, par un philhellénisme romantique, religieux, libéral, mondain, la participation de la France a été multiple et décisive. Outre les autres expressions de ce mouvement - aide financière, quêtes des femmes, organisation de concerts, campagne de presse, discours de tribune, livres, brochures politiques ou historiques -, la voix des poètes du peuple s'est aussi élevée, pour défendre le droit à la liberté des révoltés de Grèce.

Ceux de Pologne contre le joug russe en 1830-1831, bien qu'ils ne reçoivent pas le soutien demandé à Louis-Philippe, remportent des victoires à Ostrolenka. Néanmoins, l'armée russe avec Paskévitch envahit Varsovie où des massacres ont lieu. La Pologne vaincue connait une période de violence et de panslavisme. Dans le même cadre d'assistance fraternelle s'insère le mouvement des Français - qui ont encore fraiche à

○ Université d'Athènes, département de littérature et de langue françaises. 
l'esprit leur intervention salutaire en faveur des Grecs - pour la liberté des Polonais contre les régimes tyranniques instaurés après le Congrès de Vienne, et plus particulièrement le despotisme tsariste. L'insurrection de novembre 1830, exprimant le refus du peuple polonais d'obéir aux ordres donnés par le tsar de marcher contre la France et la Belgique, accentue à partir de la Monarchie de Juillet ce courant polonophile, qui retentit jusqu'aux évènements révolutionnaires de 1848. Les arguments en faveur d'une politique gouvernementale prudente ont peu de poids devant la passion des poètes et des chansonniers rêvant d'affranchir les peuples opprimés de Grèce puis de Pologne. Il ne s'agit pas de compassion mais de politique.

\section{Chansons et poésies philhelléniques}

Sous forme de multiples brochures vendues au profit des Grecs, ce mouvement de solidarité élabore des documents précieux, moins par la valeur de l'expression littéraire, si on excepte les créations des maitres romantiques, que par l'ampleur de sa production et par le rôle qu'il a joué au niveau de la politique française et européenne. Ces pièces animées par les idées de la Révolution, qui dans la plupart des cas manquent de talent, de tempérament poétique original, suivent l'opinion publique et se mettent au service de l'idéal d'affranchissement de la Grèce. Leurs auteurs ont conscience, d'une part, comme apôtres des droits de l'homme, d'un devoir vis-à-vis de la liberté des peuples et, d'autre part, comme héritiers de la civilisation grecque ancienne, d'une sorte de dette à l'égard de la Grèce considérée comme la terre sacrée, le berceau de la culture occidentale.

L'écho des évènements de Souli, des massacres de Scio, de la destruction d'Ipsara ou Psara, de la mort de Lord Byron en Grèce et surtout de la prise de Missolonghi, retentit dans la presse et la poésie françaises. Ce sont les faits culminants de l'insurrection grecque, qui aboutit à la victoire de Navarin remportée par les escadres de la triple alliance réalisée entre les Français, les Anglais et les Russes, sur la flotte turco-égyptienne, le 20 octobre 1827. Cette victoire, qui a assuré la liberté des Grecs, peut être considérée comme un résultat du mouvement de solidarité qui a soulevé les peuples européens.

Il est naturel que la révolution grecque de 1821 ait attiré la méfiance et la réaction des politiciens de la "Sainte Alliance ». Ils ont pourtant dû suivre le mouvement. Leur politique a subi l'influence du courant philhellénique et opéré un changement radical, dont le fruit a été l'appui déter- 
minant apporté aux Grecs à Navarin. En France, le philhellénisme avait le plus pesé « sur le gouvernement réfractaire, l'amenant peu à peu, à force de pression, à s'agréger à la triple alliance $»{ }^{1}$. Ce mouvement y touche toutes les classes : les libéraux inspirés par la révolte contre l'oppresseur, des monarchistes qui opposent les Grecs chrétiens aux Turcs musulmans, la Croix au Croissant, les intellectuels, les mondains, les femmes etc. ${ }^{2}$. Dans la préface de son œuvre poétique, Pierre Lebrun présente la victoire au combat naval de Navarin comme « une bataille gagnée par les peuples qui se donnent la main les uns aux autres au nom de la liberté et de la religion [...], bataille qui proclame l'avènement triomphal de cette puissante opinion publique, assise au-dessus des trônes vraiment reine pour la première fois, disposant des canons et des flottes, commandant aux amiraux; entrainant les souverains eux-mêmes et les forçant, pour ainsi dire, à légitimer sa victoire et à adopter ses lauriers $\gg{ }^{3}$.

L'hellénisme classique a constitué la source initiale de l'opinion publique philhellène ${ }^{4}$, ainsi que les impressions des voyageurs soulignant (avec les charmes exotiques du pays) les misères des chrétiens esclaves. Cette filiation et cette fraternité conduisent les poètes à l'impression d'avoir envers la Grèce une sorte de "dette sacrée ». Témoin, J.-P. G. Viennet :

En arrachant ce peuple au sceptre des tyrans,

Soyez ses alliés et non ses conquérants;

Acquittez noblement cette dette sacrée

Qu'imposa son génie à l'Europe éclairée.

Héritiers de ses arts, héritiers de ses lois

Rendez-lui ses bienfaits, rendez-lui tous ses droits ${ }^{5}$.

Nourris de cet hellénisme sentimental, se trouvent au premier rang du mouvement philhellénique de grands poètes, tels V. Hugo, A. de Lamartine, G. de Nerval, A. de Vigny, C. Delavigne, Béranger, A. Guiraud,

1. G. Isambert, 1900, L'Indépendance grecque et l'Europe, Paris, Plon-Nourrit et Cie, p. 322.

2. R. Canat, 1911, La Renaissance de la Grèce antique (1820-1850), Paris, p. 10.

3. Le Voyage de Grèce, 1828, Paris, Ponthieu et Cie, p. XV, XIX. Voir aussi F. TabakiIona, 1997, " Voyage et production littéraire-Souvenirs de voyage en Grèce » d'Ernest Fouinet, "Voyage de Grèce » de Pierre Lebrun, dans Actes du Colloque International Le Voyage aux $18^{e}$ et $19^{e}$ siècles, Athènes, p. 131-143.

4. A. Dimopoulos, 1962, L'Opinion publique française et la Révolution grecque (18211827), Imprimerie Idoux, Nancy, 1962, p. 20.

5. J.-P. G. Viennet, 1821, Épitre aux rois de la Chrétienté sur l'indépendance de la Grèce, suivie de l'épitre à Morellet sur la philosophie du $18^{\mathrm{e}}$ siècle, Paris, Ladvocat. 
A. Tastu, etc., qui s'insèrent très tôt dans le philhellénisme, et aussi une foule d'autres poètes, des écrivains mineurs et des chansonniers. Les textes de la Note sur la Grèce de Chateaubriand et l'Appel aux nations chrétiennes en faveur des Grecs de Benjamin Constant constituent des brochures de base, qui ont joué un rôle important dans le mouvement en tant que plaidoyers en faveur des Grecs. Des pièces poétiques célèbres comme Héléna et Sur la Mort de Byron, d'Alfred de Vigny, Le Dernier Chant du pèlerinage d'Harold, Invocation pour les Grecs, d'Alphonse de Lamartine, Les Orientales, À Canaris de Victor Hugo, Ipsara de Gérard de Nerval, Les Grecs, épitre au grand-Turc de Barthélemy ${ }^{6}$, s'en inspirent pour illustrer à la romantique la cause des Grecs, avivant la compassion vis-à-vis de leurs souffrances et l'enthousiasme pour leur courage. La mort glorieuse de Lord Byron suscite une vague d'admiration parmi les poètes français. En phase avec l'opinion des masses, ils protestent contre l'indifférence du pouvoir, de l'Église, des souverains européens qui laissent détruire ce peuple frère. Les vers de Hugo sont révélateurs de l'indignation qui sous-tend leur appel à une intervention :

[...] En vain pour te sauver, patrie illustre et chère,

Nous réveillons le prêtre endormi dans sa chaire,

En vain nous mendions une armée à nos rois ${ }^{7}$.

En Grèce! en Grèce! adieu, vous tous! il faut partir!

Qu'enfin, après le sang de ce peuple martyr,

Le sang vil des bourreaux ruisselle!

En Grèce, ô mes amis! vengeance! liberté !

Ce turban sur mon front! ce sabre à mon côté!

Allons! ce cheval, qu'on le selle! ${ }^{8}$

Des concours poétiques sont organisés par des Académies ou par la presse périodique; des odes, des épitres, des chants, des chansons, de la part de poètes qui prennent la lyre pour la première fois, des vers de femmes aussi payent le tribut poétique au courage sublime des combattants. Le nombre de ces pièces, la dimension de la fécondité poétique à ce

6. Épitre parue anonyme chez les marchands de nouveautés, avec la date du 26 avril 1826, puis signée Barthélemy dans l'édition Dénain et Perrotin de ses Euvres en 1831. Cf. Caminet Darbousset St., "Auguste Marseille Barthélemy, poète militant», Annali dell'Instituto Universitario Orientale, 35 (2), Naples, p. 358.

7. «Navarin », dans Les Orientales, Euvres poétiques, Paris, Gallimard, NRF, « Bibliothèque de la Pléiade », 1964, tome 1, p. 614.

8. «Enthousiasme », ibid., p. 605. 
sujet, sont évoqués dans la presse de l'époque : « J'offre à parier, écrit un journaliste, que le nombre des odes seulement surpasse déjà le nombre des soldats armés que les Grecs sont en état d'opposer à leurs cruels oppresseurs ${ }^{9}$. Des Français, renonçant aux vœux stériles, désirent depuis le début de la révolution, s'engager dans cette guerre. À seize ans, Nerval se désole : "Faible enfant, je ne puis que verser quelques larmes, Homme, peut-être, un jour je saurai les venger $»{ }^{10}$.

D'où l'importance des genres «appel au peuple» et «chant du départ ». L'enthousiasme de multiples anonymes pour s'engager dans la lutte pour l'indépendance, exiger le recours aux armes pour défendre la cause d'une liberté qui devient aussi la leur, trouve son expression, par exemple, dans une chanson intitulée Le Départ pour la Grèce :

\author{
Que pour la Grèce des esclaves \\ Soupirent des stériles vœux, \\ On verra les armes des braves \\ Protéger ses champs malheureux. \\ Dans une honteuse indolence \\ Que le monde reste arrêté! \\ La cause de la liberté \\ Sera la cause de la France ${ }^{11}$.
}

Les massacres de Scio laissent l'Europe sans réaction, et ces bardes du peuple, déçus, de démasquer les intentions des grandes puissances. L'anonyme P. Ch., entre autres, adresse aux Hellènes ces vers de désillusion :

Pour fonder votre état et votre indépendance

Autant que vous pourrez n'ayez recours qu'à vous :

Les grands vendent souvent bien cher leur assistance

Et se ressemblent tous ${ }^{12}$.

Béranger lui-même ne se contente pas de chansonner à la mode. Outre ses créations philhelléniques qui reflètent l'opinion de ses contemporains libéraux : Voyage imaginaire, Le Pigeon messager, L'Ombre d'Anacréon, il adresse aux puissants des vers engagés, pleins d'amertume, de reproche,

9. Le Miroir, 9 déc. 1821.

10. G. de Nerval, 1989, Euvres complètes, Paris, Gallimard, NRF, « Bibliothèque de la Pléiade $»$, p. 4-8.

11. Anonyme, Le Départ pour la Grèce, air de Colonne, Le Miroir, 26 juin 1822.

12. P. Ch., 1822, Un Chant poétique sur les massacres de Scio, Paris, Delaunay et Chaumerot, p. 5. 
au nom de l'humanité outragée, ainsi dans sa chanson Psara, Chant de victoire des Ottomans. Les Turcs y sont représentés criant : "Pillons ces murs! de l'or! du vin! des femmes! [...] Les rois chrétiens ne vous vengeront pas $»^{13}$.

J.-E. Gautier, à l'occasion des désastres de l'ile de Psara, prend l'initiative de plaider auprès du Souverain Pontife la cause du peuple grec et lui suggère que l'Église d'Orient reconnaissante puisse se soumettre « au triple diadème » et consentir à l'union des Églises, rappelant en même temps aux rois chrétiens leurs devoirs sacrés :

Et vous, ô Pontife suprême,

Vous, père des Chrétiens, prêtez-leur vos secours :

Que le Grec délivré vous bénisse et vous aime;

$[\ldots]$

Eh! que vous prescrit donc cette sainte alliance

Que vous avez jurée, ô Rois!

Si ce n'est du malheur d'embrasser la défense?

Rompez les fers du Grec, et rendez-lui ses droits ${ }^{14}$.

La campagne politique dirigée vers les gouvernants s'amplifie au niveau populaire. Des élégies, des dithyrambes, des " hellénides », des " athéniennes », des « helléniennes » s'inspirent de l'héroïsme des Grecs. Innombrables, les versificateurs aux noms plus ou moins connus qui s'y exercent: J. Pernier, A. Muffle, J.-P. Levée, P. Andrien, A. Nicolas, E. Michelet, Pellet, Cl. A. Dorion, D. Adolphe, A. Bétourné, A. Montémont, chantre du général Fabvier, héros de l'indépendance grecque ${ }^{15}$, etc.

La vague sera aussi profonde moins de dix ans plus tard. C'est à un second mouvement de solidarité européenne, accompagné de manifestations populaires et d'un soulèvement poétique manifesté par de nombreuses chansons de rue, qu'on assistera en France. Il s'agit cette fois de solidarité envers la Pologne. Les mêmes termes et images reviennent: sauver, délivrer, renverser la tyrannie, briser les chaines, les fers, le joug, suivant les mêmes schémas : déploration, indignation, exhortation, désir d'agir, appel au Roi puis à la République, autour du même mot-thème de liberté.

13. P.-J. de Béranger, 1857, Euvres complètes, Paris, Perrotin, tome 2, p. 123.

14. Ipsara, Chant élégiaque, 1824, Paris, Le Normant fils, p. 9-10.

15. Voir F. Tabaki-Iona, 1993, Poésie philhellénique et périodiques de la Restauration, Athènes, Société des Archives Helléniques, Littéraires et Historiques, p. 101. 


\section{Chansons et poésies pour la Pologne}

Après les destructions et les massacres des Russes à Varsovie et la défaite des Polonais, l'opinion publique française commence à s'émouvoir. En réponse au Manifeste polonais du 20 décembre, un Comité Central de Secours à la Pologne présidé par Lafayette lance un appel, signé par Hugo, Béranger et Delavigne, qui révèle une prise de position enthousiaste du peuple français à l'égard de la cause polonaise. Dans cet appel apparaissent des allusions à l'apport de l'aide française aux Grecs et particulièrement à la contribution des femmes :

La Pologne a appelé la France à son secours et celle-ci a répondu à cet appel. [...] La politique des gouvernements peut avoir ses droits et ses lois, mais la conscience des peuples ne connait qu'un seul droit, la liberté des peuples, une seule loi, l'aide des opprimés. [...] Femmes françaises, apportez votre appui aux femmes polonaises. Il n'y a pas si longtemps, toute l'humanité vous a cordialement remerciées pour votre dévouement, l'esprit avec lequel vous aviez su le rendre plus efficace; aujourd'hui l'héroïsme vous supplie de donner des dons patriotiques. Les Grecs vous bénissent et la Pologne vous devra partiellement son indépendance et ses libertés ${ }^{16}$.

Le parallélisme établi entre le sort de la Grèce et celui de la Pologne se réalise chez Alfred de Musset avec l'image renouvelée d'un sacrifice sanglant, d'un nouveau Missolonghi, qui seul, selon lui, pourra bouleverser l'opinion publique et tous les «blasés » du vieux continent :

Jusqu'au jour, ô Pologne! où tu nous montreras Quelque désastre affreux, comme ceux de la Grèce, Quelque Missolonghi d'une nouvelle espèce, Quoi que tu puisses faire, on ne te croira pas ${ }^{17}$.

Aspirant à soulever les autres peuples dans une coalition contre les Russes, un grand nombre de poètes du peuple, surtout des chansonniers républicains, chantent le sort déplorable des Polonais et flagellent la tyrannie des Cosaques. La révolution de 1848 ne va pas s'opérer en vase

16. Appel reproduit par Le Polonais consciencieux du 27 janvier 1831. Voir aussi La Pologne et la France en 1830-1831, Paris, Le Comité Central en faveur des Polonais, 1831, p. 1-18.

17. Poésies Complètes, 1957, Paris, Gallimard, NRF (coll. «Bibliothèque de la Pléiade »), p. 491. Écrits en 1831, ces vers À la Pologne sont publiés dans Le Magasin de librairie du 10 décembre 1859 et recueillis dans la première édition des Euvres posthumes, 5. Poésies complètes, p. 857. 
clos. Le rêve de la liberté se réanimant, ces chansonniers appellent les Français à l'action, en leur rappelant l'intervention efficace en faveur de l'indépendance de la Grèce. Les vers suivants des chansons de Loynel et de Rochefort, presque vingt ans après cette intervention, gardent le souvenir de la solidarité française et européenne à l'égard des Grecs, qui comme un phare éclaire les attitudes à adopter pour d'autres peuples souffrant de tyrannie. Le parallélisme entre la lutte des Grecs et celle des Polonais s'établit pour une même cause, la liberté :

Oh! comme toi, la Grèce à l'agonie

Avait osé rêver la liberté!

Pour renverser l'ignoble tyrannie

Qui l'opprimait, notre bras fut prêté, Pologne, à nous, notre vieille alliée, À nous bientôt de remplacer nos torts. De tous les cœurs tu n'es pas oubliée, La liberté bénira nos efforts... ${ }^{18}$.

Rappelez-vous que le Péloponnèse

Sut s'affranchir d'un pouvoir détesté, Les fils des Grecs tombaient dans la fournaise, Mais ils mouraient au cri de liberté!

De leurs tombeaux sortit une patrie, Des lois, un peuple et d'immortels succès !... Imitez-les, enfants de Cracovie, Rendez la gloire Et la victoire À l'aigle polonais ${ }^{19}$.

Ce n'est pas seulement la lutte des Grecs contre la tyrannie qui inspire Hugo, Béranger, Vigny, Delavigne, Népomucène Lemercier, etc., c'est l'état même des Polonais insurgés. Outre les souscriptions publiques, les concerts, les représentations théâtrales, des chantres de leur cause comme Auguste Barbier, Barthélemy, Émile Debraux, Maurice de Guérin, Joseph Roumanille, Marcelline Desbordes-Valmore, Louise Colet, font en vers ardents l'éloge du combat polonais ${ }^{20}$. Victor Hugo, dans un poème du

18. A. Loynel, « À la Pologne », dans Le Républicain lyrique, 2, aout 1848.

19. Rochefort, L'Aigle des Polonais (air de Liberté sainte après trente ans d'absence), 1846, Fonds de la Bibliothèque Nationale de France (BN), cote Ye 7185 (145).

20. Voir Fr. Rouquette, 1919, La Pologne et nous, L'amitié polonaise dans notre littérature, Paris, Chapelot; J. Lorentowicsz, 1935-1941, La Pologne en France (vol. 1 
12 septembre 1835, décrit la Pologne comme une femme, esclave vaincue, prête à mourir; sœur de la France, elle attend une aide de sa part, et cette aide tarde à venir, vieille ritournelle populaire :

[...] Pâle et sur le pavé tombée à deux genoux,

Triste Pologne! hélas! te voilà donc liée,

Et vaincue, et déjà pour la tombe pliée![...]

Levant tes bras meurtris et ton front qui chancelle

Et tes yeux que déjà la mort semble ternir,

Tu dis : France, ma sœur! ne vois-tu rien venir? ${ }^{21}$

Béranger, ému par ces élans de générosité, compose la chanson Poniatowski, très connue à l'époque - où, en évoquant cette figure héroïque du passé, il célèbre la fidélité du peuple polonais - et, en contrepoint, une autre chanson adressée aux Français : Hâtons-nous; on y remarque la même exhortation que dans le cas des Grecs : secourir les victimes. C'est la même expression d' " arracher le peuple à » la mort qui revient dans ses vers, avec le même sentiment désintéressé de solidarité :

Ah! si j'étais jeune et vaillant

Vrai hussard, je courrais le monde,

Retroussant ma moustache blonde,

Sous un uniforme brillant.

Le sabre au point et bataillant.

Va mon coursier, vole en Pologne;

Arrachons un peuple au trépas! ${ }^{22}$

À l'opposé de l'esprit laïque qui présidait aux chansons de la Révolution de 1789, dans les chansons populaires, en particulier celles consacrées à la Pologne, on espère l'intervention de Dieu dans le changement du destin des peuples. Il n'est pas jusqu'à ce voltairien de Béranger qui ne l'invoque, à sa manière: dans la dernière strophe de Hâtons-nous, on

Littérature-théâtre-beaux arts), Essai d'une bibliographie raisonnée, Paris, ChampionInstitut d'Études Slaves, p. 105-122; Ch. Sénéchal, 1937, La Pologne de 1830 à 1846 dans la poésie romantique française, Paris, Séances et travaux du Centre d'Études Polonaises; E. Marek, 1994, Quand toute la France devint polonaise. L'Insurrection polonaise de novembre 1830 et l'opinion française (conférence donnée le 3 juillet 1980), Lille, Études et Travaux du Club Polonia-Nord, Lille.

21. Les Chants du crépuscule, IX, Euvres poétiques, Gallimard, NRF (coll. «Bibliothèque de la Pléiade »), 1964, tome 1, p. 847-848.

22. P.-J. de Béranger, Euvres complètes, tome 2, p. 270. 
attend du geste métaphysique effectué par le poète à la place de Dieu le miracle qui sauvera la Pologne...

Si j'étais un jour, un seul jour, Le Dieu que la Pologne implore, Sous ma justice avant l'aurore, Le czar pâlirait dans sa cour : Aux Polonais tout mon amour! Je saurais trompant les oracles, De miracles semer leurs pas. Hélas! il leur faut des miracles! Hâtons-nous : l'honneur est là-bas ${ }^{23}$.

Dieu est, en effet, très souvent invoqué par les chansonniers pour approuver la juste lutte des Polonais. Selon eux, « Un tel arrêt, Dieu ne l'a point dicté ${ }^{24}$. «Aux pieds de Dieu dont la justice est grande », les droits de la Pologne figurent, balance en main; un poète anonyme écrit qu'il pèsera ces droits, surtout celui de la liberté qui sera attribuée à ce « peuple héros ».

Le même poète adresse des menaces au tsar, aux tyrans, au moyen des images du sang versé pour féconder des héros et des heures qui s'envolent rongeant les fers de l'esclavage ${ }^{25}$. Écho de cet anti-tsarisme ambiant : dans sa pièce poétique Wanda, suivie du sous-titre "Histoire russe", Vigny exprime d'une voix véhémente sa condamnation du despotisme de l'empereur russe. C'est avec la même intention que, après les Messéniennes consacrées au peuple grec, Casimir Delavigne écrit La Varsovienne. Seule la forme change : empruntant les formes du chant populaire composé de strophes en vers inégaux et suivies d'un refrain, il exprime les sentiments fraternels qui attachent la France, sensible à la lutte pour la liberté, au peuple polonais. Ces vers donnent une place importante dans la sensibilisation des Français à la solidarité européenne. Cette pièce a été composée pour un concert au profit de la Pologne ( $1^{\text {er }}$ mars 1831). On l'a chantée par la suite dans les rues et sur scène. Elle donne lieu à plusieurs adaptations polonaises et inspire plusieurs poètes en Pologne même ${ }^{26}$.

23. Ibid., p. 271.

24. Écrit par un chansonnier qui se présente comme l'auteur de la Marseillaise $d u$ Polonais dans la pièce intitulée France et Pologne ou les Orphelins de Cracovie! (air de T'en souviens-tu?), 5e éd., chez Madame Delavigne, 1846, B.N., cote Ye 7185 (153).

25. Id., Liberté défends tes droits, Chant Polonais (air du Rocher de Saint-Avelle), $5^{\mathrm{e}}$ édition, chez Madame Delavigne, 1846, ibid., cote Ye 7185 (153).

26. Voir E. Malek, 1994, Quand toute la France devint polonaise..., Lille, Études et travaux du Club Polonia-Nord, p. 36-37. 
Dans l'extrait qui suit, on entrevoit la tradition chansonnière révolutionnaire : des métaphores traduisent le mouvement, l'élan enthousiaste du départ, enrichies de sensations auditives, clameurs et sons guerriers du tambour et du clairon. Le thème de la contribution des femmes y est aussi développé :

Allons guerriers, un généreux effort!

Nous les vaincrons; nos femmes les défient.

Ô mon pays, montre au géant du nord

Le saint anneau qu'elles te sacrifient.

Que par notre victoire il soit ensanglanté;

Marche, et fais triompher au milieu des batailles

L'anneau de fiançailles

Qui t'unit pour toujours avec la liberté!

Polonais à la baïonnette!

C'est le cri par nous adopté;

Qu'en roulant le tambour répète;

À la baïonnette!

Vive la liberté!

[...] Sonnez clairons! Polonais à ton rang

Suis, sous le feu, ton aigle qui s'élance ${ }^{27}$.

Pologne et Polonais en deviennent des noms-symboles, des signes de ralliement. Avec la Fin de la Pologne du chansonnier Pierre Dupont, on ne peut manquer de mentionner Félicité Lamennais, qui rend hommage aux combattants dans son Hymne à la Pologne ${ }^{28}$. Maurice de Guérin, attribuant à la Pologne la figure de la prosopopée de la femme-sœur, s'adresse ainsi aux rois d'Europe :

Rois il faut prononcer, la Pologne chancelle

Sa force défaillit, son pied est ébranlé

Sur le sabre déjà le vainqueur essoufflé

La tient sur ses genoux, et l'étreinte est mortelle ${ }^{29}$.

Il en est de même pour les noms de Varsovie et, en 1848 surtout, de Cracovie, ne serait-ce que dans les titres. Auguste Barbier écrit « Varso-

27. C. Delavigne, 1842, Messéniennes et chants populaires, Berlin, A.D.M.T. Schlésinger, p. 85-87.

28. Avignon, Pierre Chaillot, 1832.

29. « Sur la Pologne », Poèmes, Poésies, tome 1, Société les Belles Lettres, Paris, 1947, p. 59. Voir aussi la pièce « Les deux anges », p. 80-82. 
vie ", poème qui fait partie du recueil Iambes et poèmes. Dans le style pamphlétaire de Némésis, Barthélemy compose les poèmes «Le Seize aout de Varsovie » et «Vendredi soir, seize septembre, Varsovie ». À la veille de la révolution de 1848, l'air de la Marseillaise fait chanter dans la rue des pièces comme La Polonaise, œuvre postume de J. A. Gardy, La Marseillaise du Polonais ${ }^{30}$ et la $2^{e}$ Cracovienne, Marseillaise des Cracoviens qui empruntent à l'hymne national français des isotopies, comme la liberté, la tyrannie, l'amour de la patrie, le triomphe des braves guerriers, la jeunesse qui suit la voie tracée par la vertu des ainés, à la façon de jeunes Spartiates. On y reprend aussi des éléments morpho-syntaxiques, tels que l'emploi de l'impératif, le tour interrogatif, des exhortations debout!, marchons!, aux armes!, etc. Quatre chansons intitulées Cracoviennes, spécifiées par des sous-titres, sont vendues en faveur des Polonais. La vente de ces productions est annoncée en bas de la feuille volante de la $1^{\text {èr }}$ Cracovienne. La Résurrection de la Pologne; on y lit aussi la note suivante, un appel de souscription (obole citoyenne), en faveur du « second pays » et des « frères du Nord»: « Le chant a toujours été l'un des plus puissants véhicules d'une grande et noble activité. [...] En donnant ce que l'on peut, on fait ce que l'on doit. C'est pourquoi nous faisons, et de notre inspiration et des produits de la vente, une première obole citoyenne dont nous déposerons l'offrande avec celles qui nous seront remises en faveur de notre second pays et de nos hérö̈ques frères du Nord ».

Autre thème de ralliement, très sensibilisateur à Paris, l'exil de nombreux Polonais et leur arrivée en France. Un grand courant d'émigration d'une élite intellectuelle conduit 9000 d'entre eux vers les pays occidentaux, organisant une résistance dont Paris devient le pôle politique. Écho de l'indignation que soulève la politique suivie à l'égard de la Pologne, le poème «L'émigration polonaise » de Barbier constitue une attaque contre la diplomatie pratiquée. L'absence d'aide française y est violemment dénoncée. J. Cousin, Ch. Le Page, M. de Guérin, composent des vers inspirés par la même colère.

Après la chute du roi et l'avènement de la République en France, saluée avec enthousiasme en février 1848, les Français se considèrent comme le guide qui mènera l'Europe à l'ère républicaine. Le 25 février,

30. L. C., La Marseillaise du Polonais précédée de La Pauvre Pologne, Paris, Mme Delavigne, 1846, B. N., cote Ye 7185 (147). 
les Polonais de Paris publient un manifeste pour se rassembler : le lendemain, ils sont 2000 à fraterniser avec la population parisienne et se rendre à l'Hôtel de Ville. Néanmoins, depuis les évènements qui suivent l'insurrection de Cracovie, les appels lancés par des Polonais exilés et ceux qui sont adressés au gouvernement provisoire de 1848 n'arrivent pas à faire dévier la politique de paix de Lamartine, qui permet la formation d'une légion polonaise mais sans lui apporter d'appui officiel. Malgré ses sentiments fraternels à leur égard, il ne prend position que pour la paix en Europe comme le révèlent ses projets exprimés dans son discours du 2 mars ${ }^{31}$. Les Polonais à Paris, avec leur compatriote Wolowski représentant élu, obtiennent du Gouvernement Provisoire que l'Assemblée Constituante décide de discuter les affaires polonaises le 15 mai 1848. Les clubs de leur côté organisent une manifestation, une journée, pour cette date; la foule envahit l'Assemblée demandant un vote d'engagement envers les Polonais et mettant la Chambre en désordre; elle exige une décision immédiate. Le ministère des Affaires étrangères oppose des arguments politiques en faveur d'une diplomatie prudente à la rêverie généreuse du peuple parisien.

Celui-ci n'en a cure. Le lyrisme populaire brassant les images extrêmes pousse les antagonismes, dont la France forme l'extrémité lumineuse. Les chansonniers expriment la conviction générale qu'elle servira d'exemple à tous et, abusant de la métaphore de la lumière et du feu, ils qualifient les évènements de 1848 d' "étincelle » d'un incendie qui embrasera toute l'Europe ou de « cratère » révolutionnaire qui brulera les tyrans :

\section{[...] Pologne, Espagne, Italie, Angleterre Vous palpitez! L'Europe est un volcan Et notre France est son brulant cratère. Debout! l'esclave a brisé son carcan ${ }^{32}$.}

Dans les chansons suivantes prêchant la fraternité des peuples et les appelant à la lutte contre la « race assassine », l'opposition est absolue entre l'instabilité de la position royale (termes: chanceler, renverser, trembler, échiner) et la force de l'union des peuples (termes: frères,

31. A. de Lamartine, discours du 2 mars 1848. Voir Le Moniteur du 25 mars 1848 et L'École des Communes, journal des progrès administratifs, $17^{\mathrm{e}}$ année, Paris, Imprimerie et Librairie administratives, 1848 , p. 72-75.

32. V. Rabineau, « Les Vieux Tambours » (air de Bagnère, Bagnère), dans Le Républicain lyrique, 5, novembre 1848. 
destins... certains, serrer, grands, marcher, même bannière, rallier, bras déchainés). Manichéisme à la Hugo en vers de mirliton :

\author{
Frères lointains \\ Vos destins \\ Seront prompts et certains \\ Quand maint trône chancelle \\ Serrez vos rangs. \\ Peuples grands \\ Renversez les tyrans. \\ $[\ldots]$ \\ Tremblez race assassine \\ Revenez, chiens \\ D'Autrichiens \\ Cosaques et Prussiens, \\ Venez qu'on vous échine ${ }^{33}$ \\ Peuples marchons sous la même bannière \\ Ralliez-vous aux Français nos ainés, \\ Belle Pologne, à nos cœurs toujours chère, \\ Des nations les bras sont déchainés ${ }^{34}$.
}

Les liens fraternels entre les peuples étant ainsi soulignés, les frontières se trouvent mises en cause. La vague est extensible. Les peuples outragés, à l'instar de la Pologne, ceux de l'Irlande, de l'Italie, de la Hongrie, de l'Allemagne émeuvent les Français qui aspirent à briser la chaine avilissante des nations et à proclamer « la liberté de tous ». Des vers prêchent une solidarité générale et encouragent la lutte de tous ces peuples contre les tyrans. On constate, à travers les chansons, que la révolution de Février 1848 et la proclamation de la République en France déchainent un mouvement d'assistance aux nations qui luttent pour leur liberté, afin de provoquer la déchéance des dynasties par la prise de conscience des droits des peuples à disposer d'eux-mêmes. La dimension de la révolution, telle qu'elle est envisagée par les chansonniers républicains, dépasse les frontières françaises. Elle répond d'avance à ceux qui, tel Hugo, prêchent l'Europe. Nous lisons, sur un appel à l'engagement pour la liberté des peuples, ces vers dédiés à Lamartine :

33. Louvet, «Le Citoyen Mayeux en 1848 » (air de la Grande Orgie), dans Le Républicain lyrique, 4, octobre 1848.

34. C. L.- C. (Colmance) « Appel à tous les peuples » (air des Trois Couleurs), ibid., 1, aout 1848 . 
Brave Pologne qu'on oublie

Va, ta délivrance a sonné,

Et toi, jeune et chaude Italie,

Vois l'aigle double couronné.

Soulevez-vous, peuples des braves,

À notre voix brisez vos fers,

Les hommes ne sont plus esclaves!

La liberté pour l'univers! 35

Les certitudes vont plus loin. On déclare non seulement la suppression des frontières entre les peuples fraternisés mais aussi l'union en un seul état, un seul symbole de l'unité des nations, un seul drapeau, sous les accents d'une Marseillaise universelle, union réussie, dont la vie paisible a été cimentée, selon les vers, par le sang des peuples :

Plus de soldats, plus de frontières,

Peuple, plus de sanglants combats;

Plus de sanglots dans nos chaumières,

Non, plus de rois, plus d'États ${ }^{36}$.

Utopie de la fraternisation: le Polonais devient le parangon d'un peuple luttant contre un joug tyrannique et les despotes de toutes parts appartiennent, selon la muse populaire, à une " race » qui «a méprisé la Pologne $»{ }^{37}$ et exerce son "vrai métier de Cosaque $»{ }^{38}$. Deux entités sont face à face, dans une sorte de trans-histoire exemplaire. Dans la Chanson patriotique de Fresne, un émigré polonais prend la parole et signale que l'avènement de la République en France constitue une date charnière qui va déterminer le sort de tous. Cette chanson retrouve la thématique du Chant de Départ de M.-J. Chénier; on y reprend le thème du moment propice à l'éclatement de l'acte révolutionnaire, avec l'expression «l'heure a sonné », et celui du sacrifice de la vie sur l'autel de la liberté :

L'heure a sonné; Polonais, plus d'entraves

La Liberté fait trembler le tyran.

35. E. G., dédié à Lamartine, 1848, Plus de Rois ! air de Guerre aux tyrans (Charles IVopéra), B.N., cote Ye 7185 (166).

36. J. Chalory, 1848, La Marseillaise européenne et universelle (air de la Marseillaise), B.N., cote 7185 (262).

37. G. Leroy, 1848, Mémorables journées des 22, 23, et 24 février 1848 (air des Trois Couleurs), ibid., cote Ye 7185 (272).

38. L. C., 1848, Hommage à M. Michelet et aux étudiants de Paris (air de Muse des Bois), ibid., cote Ye 7185 (171). 
Sachons mourir plutôt que d'être esclaves,

Mais soutenons l'honneur de l'Aigle blanc ${ }^{39}$.

Les fers des « braves Polonais » vont être « brisés » : suffit-il pour cela que l'hymne de la liberté retentisse en France?

Réalité de la fraternisation : certes l'accueil et l'assistance de la France (« hospitalier rivage ») aux réfugiés polonais sont mis en scène dans des vers comme

Pendant vingt ans la France hospitalière

Nous accorda ses généreux bienfaits (Fresne);

$\mathrm{ou}$

Venez enfants, nous vous tendons les bras

[appel adressé aux enfants proscrits de Cracovie

par les soldats des frontières françaises];

ou encore

Aux orphelins la France tend les bras.

« Sœurs », France et Pologne se donnent le bras. À la « belle Pologne », si « chère » aux cœurs des Français, répond la « belle France ».

Mais, en contrepoint de l'utopie comme de la réalité fraternelles, les chansons concluent souvent sur une interrogation qui sonne comme une accusation. Témoin, cette prosopopée :

Ô belle France! ô ma belle patrie,

Entends la voix des braves Polonais,

Songes-y bien, la liberté chérie

Leur promettait le secours des Français.

Un cri du cœur chez nous s'est fait entendre

Laisseras-tu mourir sans les défendre

Les Polonais fils de la liberté? ${ }^{40}$

Les mots vengent l'échec. Des menaces, aussi bien que des exclamations, des cris, des appels de solidarité traduisent les sentiments fraternels envers les Polonais. Des vers anonymes personnifient une Pologne qui « vers nous tend ses bras sanglants » pour implorer le secours du peuple

39. (Air des Trois Couleurs), 1848, ibid., cote Ye 7185 (169).

40. G. Leroy, L.-Ch. Durand, «France et Pologne» (air de Rendez-moi mes vieux souvenirs), ibid., 2, aout 1848. 
français, opposant les tyrans aux esclaves à libérer, dans une antithèse entre la victoire, la vie de la Pologne libre et la mort de ses tyrans. Image archétypale, puisque « Des peuples c'est la volonté » :

Dans la Pologne ah! plus d'esclaves

Des peuples c'est la volonté.

Guerre aux tyrans! victoire aux braves

Vive à jamais la Liberté ${ }^{41}$.

Tout ce lyrisme, on le voit, porte au-delà de la Pologne. Ils s'élargit à bien d'autres peuples, au moins dans un cadre européen. Une affiche non signée couvre les murs de Paris et proclame dès mars 1848 : «N'oubliez pas la Pologne. Ce n'est pas seulement une nationalité, c'est un principe, c'est le principe de la fraternité, celui de l'indépendance des nations, que vous venez de proclamer et que vous avez à défendre ${ }^{42}$. Si l'expression « sœur polonaise » manifeste le lien particulièrement étroit ressenti par les Français, dans beaucoup d'autres chansons de cette époque, on relève des sentiments de solidarité à l'égard d'autres peuples européens, des appels à l'action, à la mobilisation, des exhortations véhémentes à s'allier sous la bannière de la liberté et de la République, contre tous les rois, ennemis sanguinaires. L'extrait ci-dessous est révélateur de cet état d'esprit :

Hongrois, Saxons, Italiens, aux armes,

Peuples marchons sous la même bannière,

Ralliez-vous aux Français, vos ainés

Belle Pologne à nos cœurs toujours chère ${ }^{43}$.

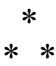

Le courant philhellénique pour l'affranchissement de la Grèce souffrant du joug ottoman, qui s'est étendu aussi à l'art poétique et chansonnier, a duré avec intensité jusqu'à la réalisation de l'objectif qu'a été la décision politique européenne suivie de l'indépendance des Hellènes. Ce mouvement favorable aux Grecs s'était emparé de toutes les couches de la

41. A. P., sous-lieutenant au $11^{\mathrm{e}}$ de Ligne, 1848, Chant patriotique (air de Charles $I V$, La France a horreur du servage), B.N., cote Ye 7185 (190).

42. Les Murailles révolutionnaires de 1848, Paris, E. Picard, s.d., tome 2, p. 449-451.

43. C.-L.C., «Appel à tous les Peuples». 
société française - même chez les monarchistes, qui étaient touchés par les tourments de leurs frères chrétiens -, chez les libéraux, les intellectuels, tous les Français émus par la démarche héroïque de ce peuple sans armée contre les Turcs. La nation hellénique combattant le tyran constituait non seulement le symbole acharné de la conquête de la liberté, à laquelle les Français et surtout les républicains étaient sensibles, mais aussi la terre sacrée de l'hellénisme classique, où la culture occidentale a fondé ses valeurs et où la France retrouvait les sources de sa civilisation.

Le mouvement en faveur de la cause polonaise n'a pas détourné les projets des gouvernants; en revanche il a recueilli un très grand nombre de chansons populaires, avec une presse abondante d'articles et de communiqués qui forment une croisade républicaine d'engagement révolutionnaire contre la stratégie du Congrès de Vienne. La lutte pour son affranchissement va de pair avec la lutte des Français pour l'amélioration des conditions sociales et politiques. D'une part, la campagne militante est menée en faveur de la Pologne en vue d'une solution diplomatique et militaire; d'autre part, elle sert à illustrer le principe de la liberté et les valeurs démocratiques pendant cette période de fermentation des idées républicaines et de montée de la critique sociale. La Pologne a été un thème-prétexte mobilisateur, un symbole pour les républicains, cristallisant des sentiments populaires de révolte. Trop dangereux sans doute, ce mouvement n'a pas abouti à une intervention guerrière, étatique, organisée comme dans le cas du philhellénisme. Sans réussir à remettre en cause l'équilibre des États et des dominances, il reste une voix de révolte, une revendication de liberté, dans la volonté exprimée de secourir les peuples proches qui souffrent de despotisme et d'injustice.

Mais que ce soit pour la Grèce ou pour la Pologne, les poètes et les chansonniers reflètent une opinion publique en mouvement, défendent les droits de l'homme et le principe de "nationalité », aux antipodes de la politique de la Sainte-Alliance. Outre leur caractère militant, ces vers écrits pendant la période du romantisme sont animés du souffle lyrique des poètes et des chansonniers émus par le sort des peuples frères. Mais on se tromperait en ne parlant que de pitié : cette émotion, cette compassion sont l'expression d'une conscience et d'une solidarité européennes en train de naitre au niveau populaire. 